\title{
Anticoagulantes Orales de Acción Directa y sus Implicancias en la Exodoncia: Una Revisión Sistemática
}

\author{
Direct Acting Oral Anticoagulants and their Implications \\ in Dental Extraction: A Systematic Review
}

Alice Cáceres R. ${ }^{1}$; Luis Areyuna C. ${ }^{1}$ \& Liberto Figueroa C. ${ }^{2}$

CÁCERES, R. A.; AREYUNA, C. L. \& FIGUEROA, C. L. Anticoagulantes orales de acción directa y sus implicancias en la exodoncia: Una revisión sistemática. Int. J. Odontostomat., 15(3):646-652, 2021.

RESUMEN: El uso de Anticoagulantes Orales de Acción Directa (ACOD) ha aumentado considerablemente en el último tiempo. En procedimientos odontológicos, como la exodoncia, es crucial un manejo óptimo de la hemostasia de pacientes bajo tratamiento con ACOD, para equilibrar el riesgo de hemorragia y tromboembolismo. Aun no existe consenso sobre el protocolo a aplicar en pacientes con ACOD sometidos a exodoncias. El objetivo fue evaluar la necesidad de suspender o continuar el tratamiento con ACOD en pacientes sometidos a exodoncia en relación con la incidencia de episodios hemorrágicos y protocolos utilizados. Se realizó una revisión sistemática en base a los estamentos PRISMA, en las bases de datos Pubmed, Wiley, Scopus. La búsqueda incluyó estudios publicados entre 2010 - 2020 en inglés, realizados en humanos, en pacientes bajo terapia con ACOD sometidos a exodoncia y que evalúan la incidencia de hemorragia en este procedimiento. Se excluyeron estudios que involucran pacientes que reciben otros tratamientos antitrombótico concomitante, o procedimientos distintos a la exodoncia. La calidad de los estudios seleccionados fue evaluada de acuerdo con la clasificación del Centro Oxford de Medicina Basada en la Evidencia. Luego de la búsqueda, en base a criterios de inclusión/exclusión, 34 artículos fueron analizados a texto completo. Trece artículos relevantes fueron seleccionados. Once participaron en la revisión final, contando con ocho estudios de cohorte, dos casos-controles y uno serie de casos. Los estudios evidencian que no es necesario suspender la terapia con ACOD en pacientes sometidos a exodoncia, se sugiere que el momento de baja concentración farmacológica puede ser utilizado a favor del tratante. Sin embargo, existe una gran diversidad de protocolos y medidas aplicadas entre estudios, por lo que es necesario realizar estudios clínicos aleatorizados controlados, para determinar un protocolo estándar en el manejo odontológico de estos pacientes.

PALABRAS CLAVE: extracción dental, anticoagulantes [acción farmacológica], inhibidores Factor Xa, dabigatrán, hemorragia postoperatoria.

\section{INTRODUCCIÓN}

Los Anticoagulantes Orales (AO) han sido indicados para el tratamiento y prevención de tromboembolismos en pacientes que padecen diferentes patologías cardíacas y pulmonares, como la cardiopatía isquémica, fibrilación auricular y pacientes con prótesis valvulares cardíacas, entre otras (Yeh et al., 2015). El uso de estos fármacos se debe a los cambios en el equilibrio hemostático entre la coagulación y la anticoagulación sanguínea, y cualquier otro cambio más significativo en este equilibrio puede causar riesgo de hemorragia o tromboembolismo (Motta et al., 2017).
La mayoría de estos fármacos actúan como Antagonistas de la Vitamina $\mathrm{K}(\mathrm{AVK})$ y su mecanismo de acción es mediante la inhibición de los factores de coagulación dependientes de la vitamina K (II, VII, IX, X) (Shi et al., 2017). Los AVK, como la Warfarina y el Acenocumarol, se consideran el tratamiento de elección para prevenir los eventos trombóticos, pero esta medicación plantea varios problemas, entre ellos la necesidad de ajustar la dosis con frecuencia y controlar el estado de coagulación de los pacientes, así como las múltiples interacciones medicamentosas y

\footnotetext{
${ }^{1}$ Estudiante Pregrado, Escuela de Odontología, Facultad de Salud y Odontología, Universidad Diego Portales, Chile..

${ }^{2}$ Profesor Asistente, Asignatura Cirugía y Traumatología Maxilofacial, Escuela de Odontología, Facultad de Salud y Odontología, Universidad Diego Portales, Chile.
}

Received: 2020-10-16 Accepted: 2021-02-18

646 
dietéticas (De la Figuera et al., 2018). En Chile el Anticoagulante Oral más utilizado es el Acenocumarol (Berkovits \& Mezzano, 2017). Ante esta situación se han introducido los Anticoagulantes Orales de Acción Directa (ACOD), Dabigatran, Rivaroxaban, Apixaban y Edoxaban, estos están diseñados para inhibir directamente los factores de cascada de la coagulación. El Dabigatrán es un inhibidor directo de la Trombina, mientras que el Rivaroxabán, Apixabán y Edoxabán son inhibidores directos del Factor Xa (Lip et al., 2016).

Los ACOD tienen varias ventajas frente a los $A \bigvee K$ ya que poseen un rápido inicio de acción, corta vida media biológica, mayor estabilidad, uso de dosis únicas y menor interacción con medicamentos, alimentos $u$ otras sustancias. Se puede prescindir de controles periódicos, lo que en teoría aumenta la adherencia al tratamiento (Berkovits \& Mezzano). En el último tiempo, los pacientes que reciben ACOD y requieren procedimientos dentales ha aumentado. Las hemorragias son comunes después de una exodoncia en pacientes que toman AO, como los AVK y los ACOD (Ockerman et al., 2019). En el transcurso de la última década los ACOD han reemplazado progresivamente a los $\mathrm{AVK}$, que fueron la única clase disponible de anticoagulantes orales por casi 6 décadas (Enríquez et al., 2019).

Muchos médicos están familiarizados con el control de la Warfarina, que se logra con la medición frecuente del índice internacional normalizado (INR). Un rango de INR terapéutico para la mayoría de los pacientes es de 2.0 a 3.0 (Kaplovitch \& Dounaevskaia, 2019). Por otra parte, los ACOD no requieren monitoreo de rutina, aunque influyen en las pruebas de coagulación más comunes como el Tiempo de Tromboplastina Parcial activada (TTPa), Tiempo de Protrombina (TP) e INR, estas son menos relevantes debido a la elevada respuesta variable vinculada tanto a condiciones instrumentales como fisiológicas (Nathwani \& Wanis, 2017; Lupi \& Rodriguez Y Baena, 2020)

Para prevenir las hemorragias, los $\mathrm{AO}$ pueden interrumpirse antes de los procedimientos dentales. La hemorragia también puede provocar una interrupción no programada de los anticoagulantes y la interrupción temporal pone a los pacientes en riesgo de eventos tromboembólicos. Por lo tanto, el manejo óptimo de los anticoagulantes es crucial para equilibrar el riesgo de hemorragia y de tromboembolismo (Ockerman et al.). Sin embargo, no hay suficientes ensayos clínicos confiables ni consenso sobre el pro- tocolo para aplicar en pacientes con ACOD sometidos a tratamiento odontológico. Problemas como la interrupción previa del ACOD, la posibilidad de recambio transitorio con heparina, el riesgo protrombótico y las posibilidades de sangrado aparecen con mayor frecuencia entre los profesionales de la odontología (Lanau et al., 2017).

El objetivo de esta revisión sistemática fue evaluar la necesidad de suspender o continuar el tratamiento con ACOD en pacientes sometidos a exodoncia, en relación con la incidencia de hemorragia, además de identificar el protocolo que se utiliza al momento de realizar este tipo de procedimiento en estos pacientes.

\section{MATERIAL Y MÉTODO}

Se realizó una búsqueda sistemática en la literatura bajo la estructura de Preferred Reporting Items for Systematic Reviews and Meta-Analysis (PRISMA) (Urrútia \& Bonfill, 2010) con el fin de permitir la reproducibilidad de la búsqueda. Se utilizó el formato PICO para las revisiones sistemáticas (Tabla I) (Manterola et al., 2013).

Tabla I. Formato de pregunta PICO.

\begin{tabular}{ll}
\hline Participantes & $\begin{array}{l}\text { Pacientes con terapia de anticoagulantes } \\
\text { orales de acción directa directa }\end{array}$ \\
\hline Intervención & $\begin{array}{l}\text { Exodoncia } \\
\text { Comparación }\end{array}$ \\
$\begin{array}{l}\text { Suspender o no los fármacos } \\
\text { Incidencia de hemorragia }\end{array}$ \\
Outcome
\end{tabular}

Búsqueda Electrónica: La búsqueda fue realizada de manera independiente por los autores en las base de datos electrónicas, PubMed, Wiley, Scopus. La búsqueda sistemática integró estudios publicados en la literatura desde el año 2010 hasta el 2020. Se realizó la última búsqueda el día 25 de agosto del 2020 . Para la búsqueda se utilizaron las siguientes palabras claves en todos los buscadores: ("oral surgery" OR "dental extraction" OR "tooth extraction") AND ("oral anticoagulants" OR apixaban OR dabigatran OR rivaroxaban OR edoxaban OR noac OR doac OR "direct oral anticoagulants" OR "new oral anticoagulants").

Criterios de inclusión: Artículos publicados en inglés, realizados en humanos, estudios que incluyan pacientes con terapia de ACOD y evalúen la incidencia de hemorragia en pacientes sometidos a exodoncia. 
Criterios de exclusión: Estudios que no cumplan con criterios de inclusión, pacientes sometidos a otros procedimientos quirúrgicos distintos a la exodoncia, estudios con pacientes que reciben otros tratamientos antitrombótico concomitante, y artículos basados en modelos animales.

Colección y análisis de datos: Las referencias identificadas se procesaron mediante EndNote X9. Luego de eliminar los artículos duplicados, dos revisores en forma independiente examinaron los artículos en relación con el título/reseña, seleccionando aquellos estudios potencialmente relevantes de acuerdo con los criterios de inclusión/exclusión para su análisis a texto completo, eliminando aquellos no disponibles en este formato. Las discrepancias entre los revisores se aclararon por discusión y acuerdo entre los autores.

Extracción y manejo de datos: Cada revisor analizó a texto completo los artículos seleccionados. En esta segunda revisión los datos fueron procesados en una tabla Microsoft Excel, se incluyeron variables documentadas de datos generales de cada artículo: año de publicación, autores, revista, resultados principales y secundarios de los estudios, y evaluación metodológica (Moreno et al., 2018). Las variables específicas de los estudios incluyeron: (1) Tipo de estudio; (2) Participantes; (3) Enfermedad previa de participantes (4) Tipo de exodoncia; (5) Anticoagulantes prescrito; (6) Exámenes previos solicitados; (7) Protocolo previo a intervención; (8) Medidas hemostáticas; (9) Incidencia Hemorragia; (10) Conclusión de artículos.

Procedimiento de análisis: Para términos de selección se define exodoncia como el acto quirúrgico que se ocupa de la avulsión de un diente del lecho óseo donde se alberga, pudiendo involucrar levantamiento de colgajo mucoperióstico, osteotomía u odontosección de la misma pieza dentaria, llevándose a cabo de la forma menos traumática posible, con un meticuloso legrado de la cavidad de extracción. Los artículos que cumplían las variables analizadas y entregaban una metodología clara del análisis de los pacientes se procesaron en una tabla de Microsoft Excel, extrayendo información relevante para su análisis final.

Evaluación de la calidad: Basado en el diseño y el contenido de los estudios seleccionados, su calidad fue evaluada de forma independiente asignando un nivel de evidencia de acuerdo con la clasificación del Centro Oxford de Medicina Basada en la Evidencia (Manterola et al., 2014).

\section{RESULTADOS}

Los resultados de la búsqueda se resumen en el diagrama de flujo, con estructura PRISMA (Fig. 1) (Urrútia \& Bonfill). Esta búsqueda arrojó 407 títulos, 75 Pubmed, 193 Wiley, 139 Scopus. Se excluyeron 98 duplicados y se leyeron 309 títulos/reseñas. 1 estudio no se consideró ya que no estaba disponible a texto completo. 34 artículos se revisaron a texto completo excluyendo: 4 artículos que asociaban procedimientos dentales distintos a la exodoncia, 7 artículos correspondientes a revisiones bibliográficas sin un análisis clínico para el fin de este estudio, 10 revisiones sistemáticas no se consideraron debido a que los enfoques de estos estudios no fueron exclusivo a evaluar la exodoncia, incluyendo otros procedimientos quirúrgicos. 13 artículos relevantes fueron sometidos a valoración del contenido analizado, 1 artículo fue excluido debido a que no presentaba una metodología clara y falta de datos para analizar en este estudio; y 1 artículo fue excluido debido a que sus datos entregados fueron promedios de datos obtenidos.

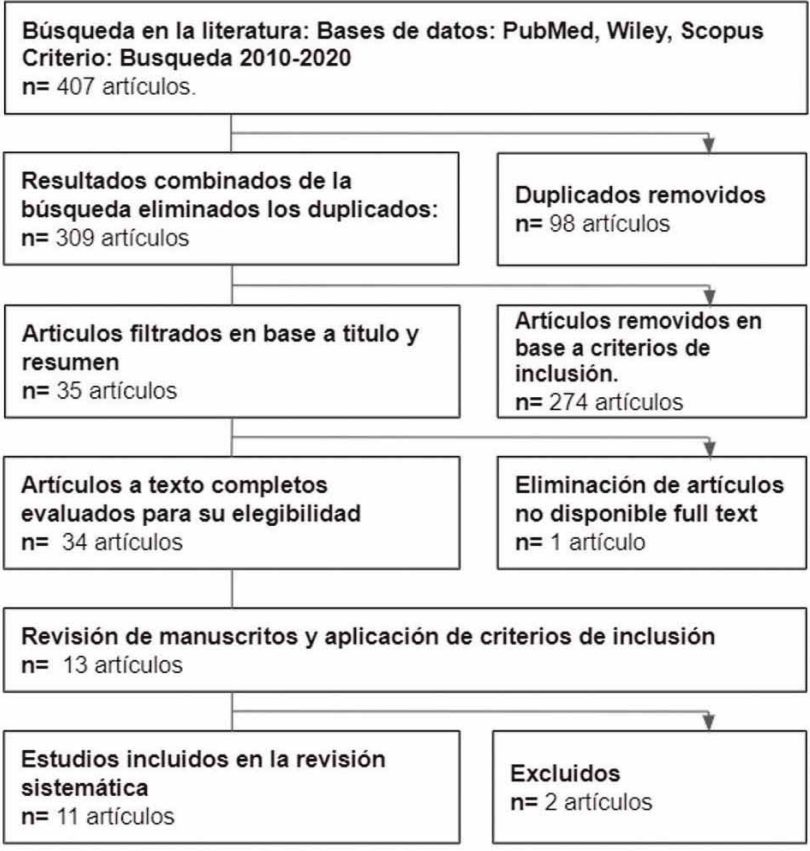

Fig. 1. Diagrama de flujo. Estrategias de selección de estudios.

Once artículos fueron seleccionados para esta revisión, contando con seis estudios observacionales prospectivos de cohortes (Miranda et al., 2016; Caliskan et al., 2017; Andrade et al. 2018; Berton et al., 2018; Yoshikawa et al., 2019; Brennan et al., 2020), 


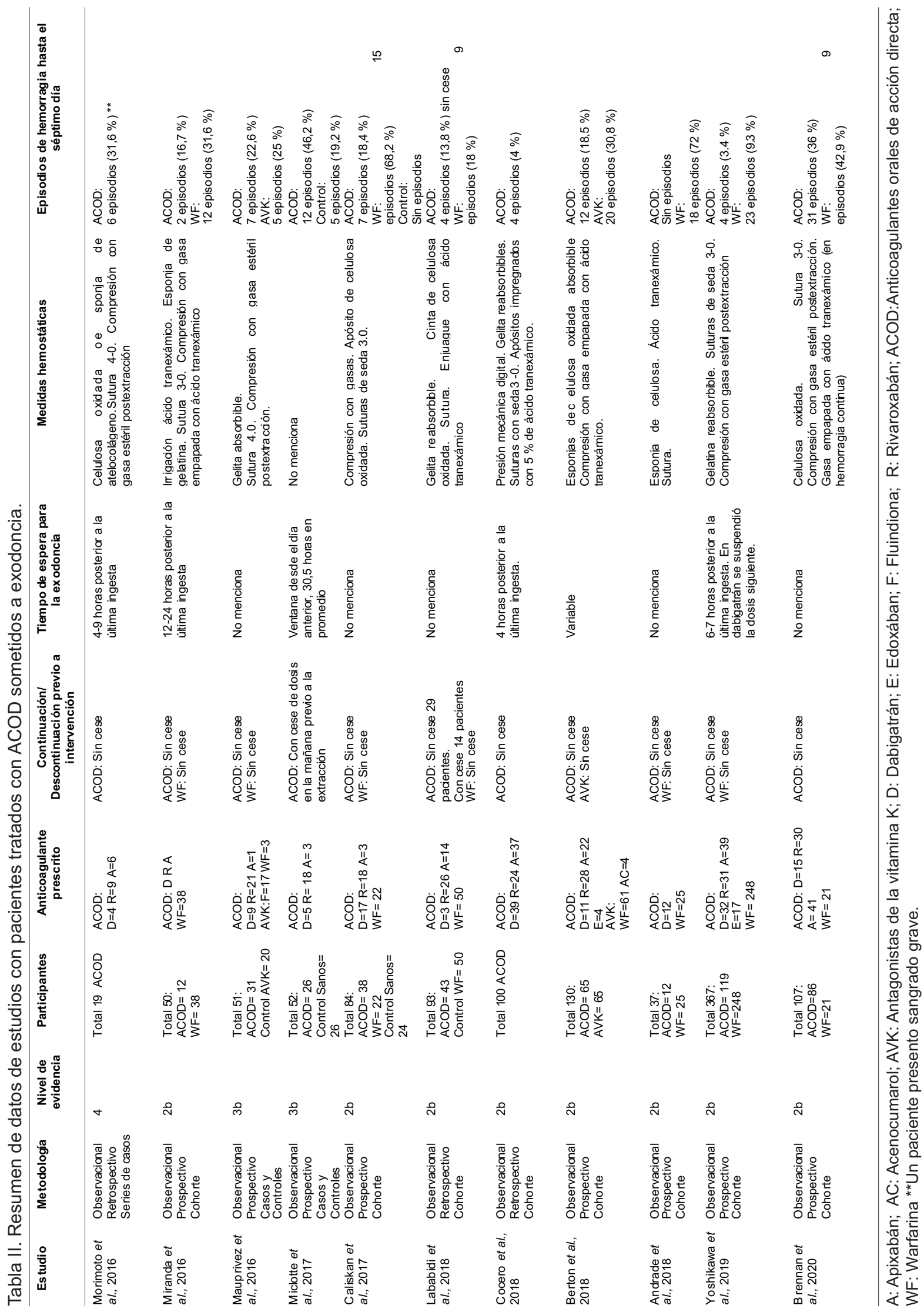


dos observacionales retrospectivos de cohorte (Lababidi et al., 2018; Cocero et al., 2018), dos observacionales prospectivos de casos controles (Mauprivez et al., 2016; Miclotte et al., 2017) y una serie de casos (Morimoto et al., 2016). El nivel de evidencia de los estudios analizados presenta ocho estudios $2 \mathrm{~b}$, dos estudios $3 \mathrm{~b}$ y un estudio 4 . Las características fueron analizadas, unificando el contenido de datos encontrados. (Tabla II).

En total se presentan 1090 pacientes, con 551 pacientes con ACOD, 489 pacientes con AVK y 50 pacientes sanos como grupo control en dos estudios. EI ACOD más utilizado por los pacientes presentes en los estudios fue el rivaroxabán, seguido del dabigatrán. En cuanto a los AVK se utilizó con mayor frecuencia la warfarina. Diez de los once artículos muestran episodios hemorrágicos en pacientes con ACOD sin cese de la medicación previa a la extracción, mientras que dos estudios analizaron los episodios de hemorragia con descontinuación de los medicamentos. Cuatro estudios utilizan un tiempo de ventana entre la ingesta de la última dosis administrada y el momento de la extracción, que va desde las 4 horas a más de 24 horas de ventana. Dentro de las medidas para favorecer la hemostasia, principalmente se utilizó gelita reabsorbible, sutura, compresión de la zona con gasa.

Los episodios hemorrágicos que presentaron los pacientes fueron principalmente leves y moderados. El promedio de episodios hemorrágicos en pacientes sin cese de medicamentos fue de $16,5 \%$ y $37,2 \%$ en pacientes con ACOD y AVK respectivamente. En el grupo de pacientes con ACOD con cese del medicamento presentó un promedio de 23,1\%. En aquellos estudios donde se contaba con un control de individuos sanos, estos presentaron un 9,6 \% de episodios hemorrágicos.

\section{DISCUSIÓN}

En cuanto a la literatura disponibles se observa una gran cantidad de estudios que entregan recomendaciones acerca del abordaje para procedimientos dentales, pero son limitados los estudios clínicos que describen la incidencia de hemorragias postoperatorias en pacientes con ACOD en comparación con pacientes sanos o con AVK (Bensi et al., 2018). Cuando un paciente anticoagulado se presenta para un procedimiento dental, el odontólogo tiene tres opciones principales: continuar la misma dosis de anticoagulación oral con agentes hemostáticos locales, disminuir la dosis o interrumpir por completo unos días antes (Chahine et al., 2019). La revisión sistemática (Manfredi et al., 2019), indica que los episodios hemorrágicos presentados en el análisis de 21 estudios que incluían pacientes sometidos a distintos procedimientos dentales invasivos bajo tratamiento con ACOD se consideran menores y manejables con medidas locales, sin necesidad de hospitalización ni transfusiones de sangre.

Dentro de las recomendaciones presentadas por los artículos analizados en esta revisión: (Caliskan et al.; Berton et al.; Brennan et al.; Lababidi et al.; Mauprivez et al.) afirman que los ACOD son fármacos seguros en términos de sangrado y las exodoncias se pueden realizar sin interrumpir o alterar el régimen anticoagulante con la ayuda de medidas hemostáticas locales. (Morimoto et al.) agrega que se debe tener un manejo cuidadoso en pacientes que presentan un mayor riesgo de hemorragia, según las consideraciones preoperatorias. (Yoshikawa et al.; Cocero et al.), recomienda que la extracción dental se realice al menos 4-6 horas después de la última dosis del ACOD para aprovechar el nivel plasmático más bajo. Por otra parte, (Miclotte et al.) recomienda que, en lugar de usar niveles mínimos, omitir la dosis matutina del ACOD es una forma conveniente de asegurar niveles bajos en plasma en el momento de la extracción, independientemente del tipo de ACOD o función renal. (Miranda et al.; Andrade et al.) señalan que el riesgo de sangrado en pacientes sometidos a exodoncia es bajo en pacientes con ACOD.

Considerando el objetivo de esta revisión, dentro del análisis de los once artículos se observa una tendencia a comparar la incidencia de hemorragia de pacientes con ACOD en relación a pacientes que utilizan AVK. La mayoría de los estudios analizaron la exodoncia sin suspender la dosis del fármaco mostrando en promedio una baja incidencia de episodios hemorrágicos en este grupo. Este promedio no es significativamente diferente al presentado en pacientes sanos. En cuanto a la gravedad de las hemorragias presentadas estas variaron entre leve a moderadas, no representando un compromiso mayor de la salud de los pacientes, dejando en evidencia que el manejo operatorio con medidas hemostáticas locales adecuadas disminuye el potencial riesgo de hemorragia en pacientes anticoagulados con ACOD.

Por otra parte, los AVK en promedio mostraron un mayor porcentaje de hemorragia $(37,2 \%)$, aun cuando las exodoncias se realizan con un INR en rango te- 
rapéutico $(2,0-3,0)$. Bajo estos resultados los ACOD muestran ciertas ventajas sobre los AVK que pueden ser utilizadas a favor del tratante al momento de la extracción dental, debido a sus propiedades farmacocinéticas y su vida media corta, varios estudios (Morimoto et al.; Miranda et al.; Yoshikawa et al.; Cocero et al.) consideran que el momento en que la concentración farmacológica baja es el indicado para realizar la exodoncia disminuyendo el riesgo de hemorragia, y siendo menos severas que en pacientes con AVK.

Este análisis indica que es seguro realizar exodoncias sin suspender el fármaco, en cuanto a la incidencia de hemorragia. Sin embargo, los datos son insuficientes para establecer un real beneficio en cuanto al manejo de pacientes con ACOD sometidos a exodoncia, debido a la diversidad en las ventanas farmacológicas utilizadas y las diferentes medidas hemostáticas utilizadas, siendo muy heterogéneas. Es por esto, que se hace necesario realizar estudios prospectivos de cohorte controlados con grupos aleatorizados doble ciego, con el fin de aclarar los riesgos y beneficios de continuar o interrumpir la terapia con ACOD en pacientes que serán sometidos a exodoncias. Para que de esta forma se pueda establecer un protocolo estándar en el manejo de pacientes que necesitan someterse a exodoncia, estando bajo terapia con ACOD.

\section{CONCLUSIÓN}

A la luz de la evidencia analizada se observa que seguro realizar exodoncia sin suspender el fármaco para evitar el sangrado postoperatorio, utilizando las medidas hemostáticas adecuadas. Sin embargo, es necesario realizar estudios clínicos aleatorizados controlados, para determinar un protocolo estándar en el manejo odontológico al momento de abordar estos casos.

CÁCERES, R. A.; AREYUNA, C. L. \& FIGUEROA, C. L. Direct acting oral anticoagulants and their implications in dental extraction: A systematic review. Int. J. Odontostomat., 15(3):646-652, 2021.

ABSTRACT: The use of Direct Acting Oral Anticoagulants (ACOD) has increased considerably in recent times. In dental procedures, such as tooth extraction, optimal management of hemostasis in patients treated with ACOD is crucial to balance the risk of bleeding and thromboembolism. There is still no consensus on the protocol to be applied in patients with ACOD in dental extraction. The aim was to evaluate the need to suspend or continue treatment with ACOD in patients submitted to dental extraction in relation to the incidence of bleeding episodes and the protocols used. A systematic review was carried out based on the PRISMA estates, in the Pubmed, Wiley, Scopus databases. The search included studies published between 2010-2020 in English conducted in humans, in patients under therapy with ACOD submitted to dental extraction and that evaluate the incidence of bleeding in this procedure. Studies involving patients receiving other concomitant antithrombotic treatments or procedures other than dental extraction were excluded. The quality of the selected studies was evaluated according to the Oxford Center for Evidence-based Medicine classification. After the search, based on inclusion/ exclusion criteria, 34 articles were analyzed in full text. 13 relevant articles were selected. 11 participated in the final review, including 8 cohort studies, 2 case-controls and 1 case series. Studies show that it is not necessary to suspend therapy with ACOD in patients who have undergone dental extraction, it is suggested that the moment of low pharmacological concentration can be used in favor of the treatment. However, there is a great diversity of protocols and measures applied between studies, so it is necessary to carry out randomized controlled clinical studies to determine a standard protocol in the dental management of these patients.

KEY WORDS: tooth extraction, anticoagulants [pharmacological action]), factor xa inhibitors, dabigatran, postoperative hemorrhage.

\section{REFERENCIAS BIBLIOGRÁFICAS}

Andrade, M.; Andrade, L.; Bispo, A. F.; Freitas, L. A.; Andrade, M.; Feitosa, G. S. \& Feitosa-Filho, G. S. Evaluation of the bleeding intensity of patients anticoagulated with warfarin or dabigatran undergoing dental procedures. Arq. Bras. Cardiol., 111(3):394-9, 2018.

Bensi C.; Belli S.; Paradiso D. \& Lomurno G. Postoperative bleeding risk of direct oral anticoagulants after oral surgery procedures: a systematic review and meta-analysis. Int. J. Oral Maxillofac. Surg., 47(7):923-32, 2018.

Berkovits, A. \& Mezzano, D. Nuevos anticoagulantes orales: actualización. Rev. Chil. Cardiol., 36(3):254-63, 2017.

Berton, F.; Costantinides, F.; Rizzo, R.; Franco, A.; Contarin, J.; Stacchi, C.; Maglione, M.; Visintini, E.; Di Lenarda, A. \& Di Lenarda, R. Should we fear direct oral anticoagulants more than vitamin $\mathrm{K}$ antagonists in simple single tooth extraction? A prospective comparative study. Clin. Oral Invest., 23(8):3183-92, 2018.

Brennan, Y.; Gu, Y.; Schifter, M.; Crowther, H.; Favaloro, E. J. \& Curnow, J. Dental extractions on direct oral anticoagulants vs. warfarin: The DENTST study. Res. Pract. Thromb. Haemost., 4(2):278-84. 2020.

Caliskan, M.; Tükel, H-C.; Benlidayi, M-E. \& Deniz, A. Is it necessary to alter anticoagulation therapy for tooth extraction in patients taking direct oral anticoagulants? Med. Oral Patol. Oral Cir. Bucal, 22(6):e767-73, 2017. 
Chahine, J.; Khoudary, M. N. \& Nasr, S. Anticoagulation use prior to common dental procedures: A systematic review. Cardiol. Res. Pract., 2019:9308631, 2019

Cocero, N.; Basso, M.; Grosso, S. \& Carossa, S. Direct oral anticoagulants and medical comorbidities in patients needing dental extractions: Management of the risk of bleeding. J. Oral Maxillofac. Surg., 77(3):463-70, 2018.

De la Figuera, M.; Cinza, S.; Marín, N.; Egocheaga, I. \& Prieto, M. A. Perfil clínico de pacientes con fibrilación auricular tratados con anticoagulantes orales de acción directa atendidos en atención primaria. Estudio SILVER-AP. Aten. prim., 50(6):359-67, 2018.

Enríquez, A.; Baranchuk, A. \& Corbalán, R. Manejo de hemorragia asociada a anticoagulantes orales directos: estado actual de las estrategias de reversión. Rev. méd. Chile.,147(1):7382, 2019.

Kaplovitch, E. \& Dounaevskaia, V. Treatment in the dental practice of the patient receiving anticoagulation therapy. J. Am. Dent. Assoc., 150(7):602-8, 2019.

Lababidi, E.; Breik, O.; Savage, J.; Engelbrecht, H.; Kumar, R. \& Crossley, C. W. Assessing an oral surgery specific protocol for patients on direct oral anticoagulants: a retrospective controlled cohort study. Int. J. Oral Maxillofac. Surg., 47(7):940-6, 2018.

Lanau, N.; Mareque, J.; Giner, L. \& Zabalza, M. Direct oral anticoagulants and its implications in dentistry. A review of literature. J. Clin. Exp. Dent., 9(11):e1346-54, 2017.

Lip, G. Y.; Mitchell, S. A.; Liu, X.; Liu, L. Z.; Phatak, H.; Kachroo, S. \& Batson, S. Relative efficacy and safety of non-Vitamin K oral anticoagulants for non-valvular atrial fibrillation: Network metaanalysis comparing apixaban, dabigatran, rivaroxaban and edoxaban in three patient subgroups. Int. J. Cardiol., 204:8894, 2016.

Lupi, S. M. \& Rodriguez Y Baena, A. Patients taking direct oral anticoagulants (DOAC) undergoing oral surgery: A review of the literature and a proposal of a peri-operative management protocol. Healthcare (Basel), 8(3):281, 2020.

Manfredi, M.; Dave, B.; Percudani, D.; Christoforou, J.; Karasneh, J.; Diz Dios, P.; Glick, M.; Kumar, N.; Lockhart, P. B. \& Patton, L. L. World workshop on oral medicine VII: Direct anticoagulant agents management for invasive oral procedures: A systematic review and meta-analysis. Oral Dis., 25(S1):157-73, 2019.

Manterola, C.; Asenjo-Lobos, C. \& Otzen, T. Jerarquización de la evidencia: Niveles de evidencia y grados de recomendación de uso actual. Rev. Chil. Infectol., 31(6):705-18, 2014.

Manterola, C.; Astudillo, P.; Arias, E,. \& Claros, N. Revisiones sistemáticas de la literatura. Qué se debe saber acerca de ellas. Cir. esp., 91(3):149-55, 2013.

Mauprivez, C.; Khonsari, R. H. Razouk, O.; Goudot, P.; Lesclous, P. \& Descroix, V. Management of dental extraction in patients undergoing anticoagulant oral direct treatment: a pilot study. Oral Surg. Oral Med. Oral Pathol. Oral Radiol.,122(5):e14655, 2016.

Miclotte, I.; Vanhaverbeke, M.; Agbaje, J. O.; Legrand, P.; Vanassche, T.; Verhamme, P. \& Politis, C. Pragmatic approach to manage new oral anticoagulants in patients undergoing dental extractions: a prospective case-control study. Clin. Oral Investig., 21(7):2183-8, 2017.

Miranda, M.; Martinez, L. S.; Franco, R.; Forte, V.; Barlattani, A. \& Bollero, P. Differences between warfarin and new oral anticoagulants in dental clinical practice. Oral Implantol., (Rome), 9(3):1516, 2016.

Moreno, B.; Muñoz, M.; Cuellar, J.; Domancic, S. \& Villanueva J. Revisiones sistemáticas: definición y nociones básicas. Rev. Clin. Periodoncia Implantol. Rehabil. Oral,11(3):184-6, 2018.

Morimoto, Y.; Yokoe, C.; Imai, Y.; Sugihara, M. \& Futatsuki, T. Tooth extraction in patients taking nonvitamin $\mathrm{K}$ antagonist oral anticoagulants. J. Dent. Sci.,11(1):59-64, 2016.
Motta, R. H. L.; Bergamaschi, C. de C.; de Andrade, N. K.; Guimaraes, C. C.; Ramacciato, J. C, Araújo, J. de O. \& LopeL, I. C. Bleeding risk in patients using oral anticoagulants submitted to surgical procedures in dentistry: a systematic review protocol. BMJ Open, 7(12):e019161, 2017.

Nathwani, S. \& Wanis, C. Novel oral anticoagulants and exodontia: the evidence. Br. Dent. J., 222(8):623-8, 2017.

Ockerman, A.; Vanhaverbeke, M.; Miclotte, I.; Belmans, A.; Vanassche, T.; Politis, C.; Jacobs, R. \& Verhamme, P. Tranexamic acid to reduce bleeding after dental extraction in patients treated with non-vitamin $\mathrm{K}$ oral anticoagulants: design and rationale of the EXTRACT-NOAC trial. Br, J. Oral Maxillofac. Surg., 57(10):1107-12, 2019.

Shi, Q.; Xu, J.; Zhang, T.; Zhang, B. \& Liu, H. Post-operative bleeding risk in dental surgery for patients on oral anticoagulant therapy: A meta-analysis of observational studies. Front. Pharmacol., 8:58, 2017.

Urrútia, G. \& Bonfill, X. PRISMA declaration: a proposal to improve the publication of systematic reviews and meta-analyses. Med. Clin. (Barc), 135(11):507-11, 2010.

Yeh, C. H.; Hogg, K. \& Weitz, J. I. Overview of the new oral anticoagulants: opportunities and challenges. Arterioscler. Thromb. Vasc. Biol., 35(5):1056-65, 2015.

Yoshikawa, H.; Yoshida, M.; Yasaka, M.; Yoshida, H.; Murasato, Y.; Fukunaga, D.; Shintani, A. \& Okada, Y. Safety of tooth extraction in patients receiving direct oral anticoagulant treatment versus warfarin: a prospective observation study. Int. J. Oral Maxillofac. Surg., 48(8):1102-8, 2019.

Dirección para correspondencia:

Luis Areyuna Cortes

Escuela de Odontología

Facultad de Salud y Odontología

Universidad Diego Portales

Ejército 233

Santiago

CHILE

E-mail: luis.areyuna@mail.udp.cl 